\title{
Correction to: Design for Teaching Surroundings Based on Human-Computer Interaction Techniques
}

Wei Feng

\begin{abstract}
Correction to:
Chapter "Design for Teaching Surroundings Based on Human-Computer Interaction Techniques"

in: A. Marcus and W. Wang (Eds.):

Design, User Experience, and Usability, LNCS 11585, https://doi.org/10.1007/978-3-030-23538-3_39
\end{abstract}

The book was inadvertently published with an incorrect version of an author's name in Chapter 39 as "Feng Wei" whereas it should have been "Wei Feng". This has been corrected in the corresponding chapter accordingly. 